\title{
Development of mobile communication systems for high-speed railway
}

\author{
Nedžad Branković, Aida Kalem, Adisa Medić \\ University of Sarajevo, Faculty of Traffic and Communications, Zmaja od Bosne 8, Sarajevo 71000, Bosnia and Herzegovina
}

\begin{abstract}
Development of high-speed railways set up challenges for new communication technologies. With the increase in speed, new requirements for communication systems have emerged that HSR requires greater reliability, capacity and shorter response time for efficient and safe operations. Mobile communication systems are crucial for the competitiveness of the railway industry and therefore have become one of the priorities addressed by the participants in the railway system to take advantage of technological opportunities to improve operational processes and the quality of provided transport services. The European Rail Traffic Management System (ERTMS) uses the Global System for Mobile Communications for Railways (GSM-R) for voice and data communication to communicate between trains and control centers. The International Railway Union is exploring new ways of communicating for high-speed railways because as speed increases this system becomes unreliable in information transmission. This paperwork presents an analysis of the evolution of communications on European railways since the usage of GSMR. In addition, an overview of the various alternative solutions proposed during the time (LTE-R, Future Railway Mobile Communication System) as possible successors to GSM-R technology is given.
\end{abstract}

Keywords: high-speed railway, GSM-R, LTE-R, mobile communication systems

\section{Introduction}

Communication technologies have always played a key role in the railway system due to its size, long distances, large number of employees and passengers, and constant mobility. Ensuring the optimal way of communication is primarily necessary to ensure the safe and efficient movement of trains, but also the general operation of the railway system in general. This importance of communication technologies has become even more important with the occurrence of high-speed trains.

GSM-R (Global System for Mobile communications Railway) is the first international mobile communication system developed for the requirements of railways. It is based on GSM mobile phone network standards but uses other carrier frequencies specifically designated for rail traffic and there are several additional functions adjusted to rail traffic. These additional functions provide the possibility of voice communication between the train operator and the control/monitoring centers of the railway traffic, as well as the transmission of the maximum permitted speeds in certain sections to the control cabin of the train.
GSM-R may be a secure platform for voice and digital communication between railway operational staff, including drivers, dispatchers, switch crew members, train engineers and station controllers.[1] It was developed in 1993 by the International Railway Union UIC (Union Internationale des Chemins de Fer) as a GSM platform for future railway telecommunications services. GSM-R is developed and used as part of the system for ERTMS standards and is based on GSM technology and EIRENE (European Integrated Railway Enhanced Network) and MORANE (MObile radio for RAilway Networks in Europe) specifications that guarantee the continuous exchange of information between stable and mobile railway facilities at speeds up to $500 \mathrm{~km} / \mathrm{h}$.[2] The main task of this technology is to ensure constant, reliable and uninterrupted communication on European railways, even at the speeds developed by superfast trains, and to simplify and harmonize signaling and communication. The benefits for railway companies and passengers are so great that the usage of the GSM-R system is one of the basic requirements of the European Union when building new railway lines or major overhauls of existing ones, and to accelerate the modernization of railway communications, the EU funds up to 85 percent of the projects. This system integrates all existing mobile radio applications on the railway and creates a platform for new 
services and applications in future development. The GSM-R system primarily ensures harmonization of the communication platform for railway interoperability and the European Rail Traffic Management System (ETCS). Together with the ETCS system, the GSM-R system forms a unique European Rail Traffic Management System (ERTMS).There are three levels of ETCS operation which use the GSM-R radio network for sending and receiving information from trains. At the first level ETCS-1, GSM-R is used for voice communication only. At the opposite two levels, ETCS-2 and ETCS-3, the GSM-R system is employed mainly for data transmission. GSM-R is extremely relevant for ETCS-2 and ETCS-3 because the train travels at accelerates to $350 \mathrm{~km} / \mathrm{h}$ and thus it's necessary to make sure continuous monitoring of the position and speed of the train. The Dutch, German, Swedish and French railways are among the first European railways that have incorporated GSM-R technology into their telecommunications system.

The GSM-R has been used successfully for several years, but it cannot meet all the requirements of the railway industry because it belongs to the second generation mobile network (2G).[3] The Mobile communications rail systems need to keep pace with the development trends of $4 \mathrm{G}$ and $5 \mathrm{G}$ mobile telecommunications technologies. Obsolescence is, therefore, a major problem for infrastructure managers and railway operators because certain spare parts may not be available on the market for 20 years or more. Maintenance of the GSM-R system after 2030 is questionable because service providers will not be able to maintain the system to the same extent, which will make operations increasingly difficult and expensive for the management infrastructure and also it will be difficult to maintain the same quality of service. Therefore, the railway industry is preparing for the transition to a new mobile system and related technologies.

In recent years, the issue of researchers in this field has been the Long Term Evolution for Railway (LTE-R) system, which provides more extensive rail services than GSM-R, such as rail video surveillance and multimedia train dispatching. [4] The LTE-R architecture is based on the wireless LTE communication standard and was developed from the existing GSM / WCDMA core network and belongs to the fourth generation mobile network. In the future, with the era of the $5 \mathrm{G}$ mobile network, 5G-based rail communication technology will become a trend and a key generation for rail-mobile communication technology.

\section{Overview of GSM-R}

\subsection{Development of GSM-R}

The International Railway Union (UIC) sought ways to harmonize rail communications in the early $1990 \mathrm{~s}$.
Many Railways have used cable networks and analog radio for voice and data communication. The need for interoperability has been a major initial force for the development of a common European standard in the field of communications. There were more than 35 different platforms in Europe, and German Rail (DB) itself had eight different analog systems.[5] The maintenance and updating of these different systems became increasingly difficult and expensive, and analog systems were not compatible with many modern communication systems. Between the two options, Terrestrial Trunked Radio (TETRA) or Global System for Mobile Communications (GSM), the European Railway Community - sponsored by the Union Internationale des Chemins de Fer (UIC) opted for GSM in the mid-'90s as a standard to replace existing analog railway radio systems because the GSM standard was commercially available in a market where its reliability had already been proven and certain modifications were needed to be adjusted to railway operations.

For the GSM-R new digital radio standard for European railways, specifications have been developed in the UIC project EIRENE (European Integrated Railway Radio Enhanced Network) which defines the requirements that railway radio communication systems must meet in order to ensure interoperability between national railways. These specifications define the requirements and configuration of the network, general requirements for mobile equipment, communication and towing vehicle equipment, control equipment, numbering plan, text messages, emergency calls, shunting equipment, etc.

After the EIRENE specification was completed, manufacturers began to engage in the very first projects. The MORANE project (Mobile radio for Railway Networks in Europe) was launched by the European Community in cooperation with the railways of Germany, Italy and France, which has been successfully completed and it has been proven that GSM can be a reliable solution in railways. Following the EIRENE and MORANE projects, UIC has launched a new project called ERTMS / GSM-R where ERTMS is a system consisting of the European Train Control System and the GSMR network as the telecommunications base. The UIC initiated the ERTMS project to bring together existing and future program developers to upgrade GSM-R specifications.[6]

The main goal of the UIC was to achieve interoperability at the level of the European Union with the help of the GSM-R system, i.e. to remove national border barriers without changing the equipment (locomotives, security methods, etc.). This would increase the safety, efficiency of rail transport and improve the cross-border interoperability of rail transport in Europe. European countries have recognized the potential offered by GSM-R technology and by the end of 2016, 56 countries had chosen GSM-R technology for their rail 
operations.[7] The Netherlands, Germany, Sweden and France were the first European countries that have implemented GSM-R technology. In addition to European countries, GSM-R- has been implemented in China and India.

\subsection{The Characteristics of the GSM-R system}

The GSM-R is a GSM system adjusted to the requirements of railways, and operates in the $900 \mathrm{MHz}$ frequency band and belongs to the second generation of mobile networks (2G). Besides the voice channel, it also contains a digital radio channel for data transmission, group calling, priority call determination, functional addressing and others for special railway functions. The European Telecommunications Standards Institute (ETSI) has allocated $4 \mathrm{MHz}$ frequency spectrum exclusively for railways by dividing them into separate frequencies for uploading and downloading data. The frequency spectrum for data upload is $876-880 \mathrm{MHz}$, and for download 876$880 \mathrm{MHz}$. In Table 1 some of the key performances of the GSM-R system are presented.

In the ETCS-1 system, GSM-R is used only for voice communication, in the ETCS-2 system, the GSM-R system is used for continuous train position monitoring and automatic train control, so data transfer is very important to ensure safety. Movement authorizations and indications of other signals are displayed in the cab. Except for a few signal indicators, it is possible to completely exclude the track signaling indication in the cab.

Table 1. Performance of GSM-R system [8]

\begin{tabular}{cc}
\hline Parameter & GSM-R \\
\hline Frequency & Uplink: $876-880 \mathrm{MHz} ;$ \\
Dandwidth & $0.2 \mathrm{MHz}$ \\
Cell Range & $8 \mathrm{~km}$ \\
Peak Spectral Efficiency & $0.33 \mathrm{bps} / \mathrm{Hz}$ \\
Data Transmission & Requires voice call \\
Mobility & connection \\
Handover Success Rate & Max. $500 \mathrm{~km} / \mathrm{h}$ \\
\hline
\end{tabular}

The exact position of each train and the direction of movement are regularly reported to the Radio Block Center (RBC) at timely intervals. The movement of the train is regularly monitored in RBC. The RBC has the main role in communications, it is connected to interlocking and to the GSM-R network, ie the mobile switching center (MSC). Interlocking provides route and tracks occupancy information that is processed in the radio block center and sent to movement authority (MA) traffic authorities via the GSM-R network, which contains base stations (BTS) located at distances of 7-15 km.

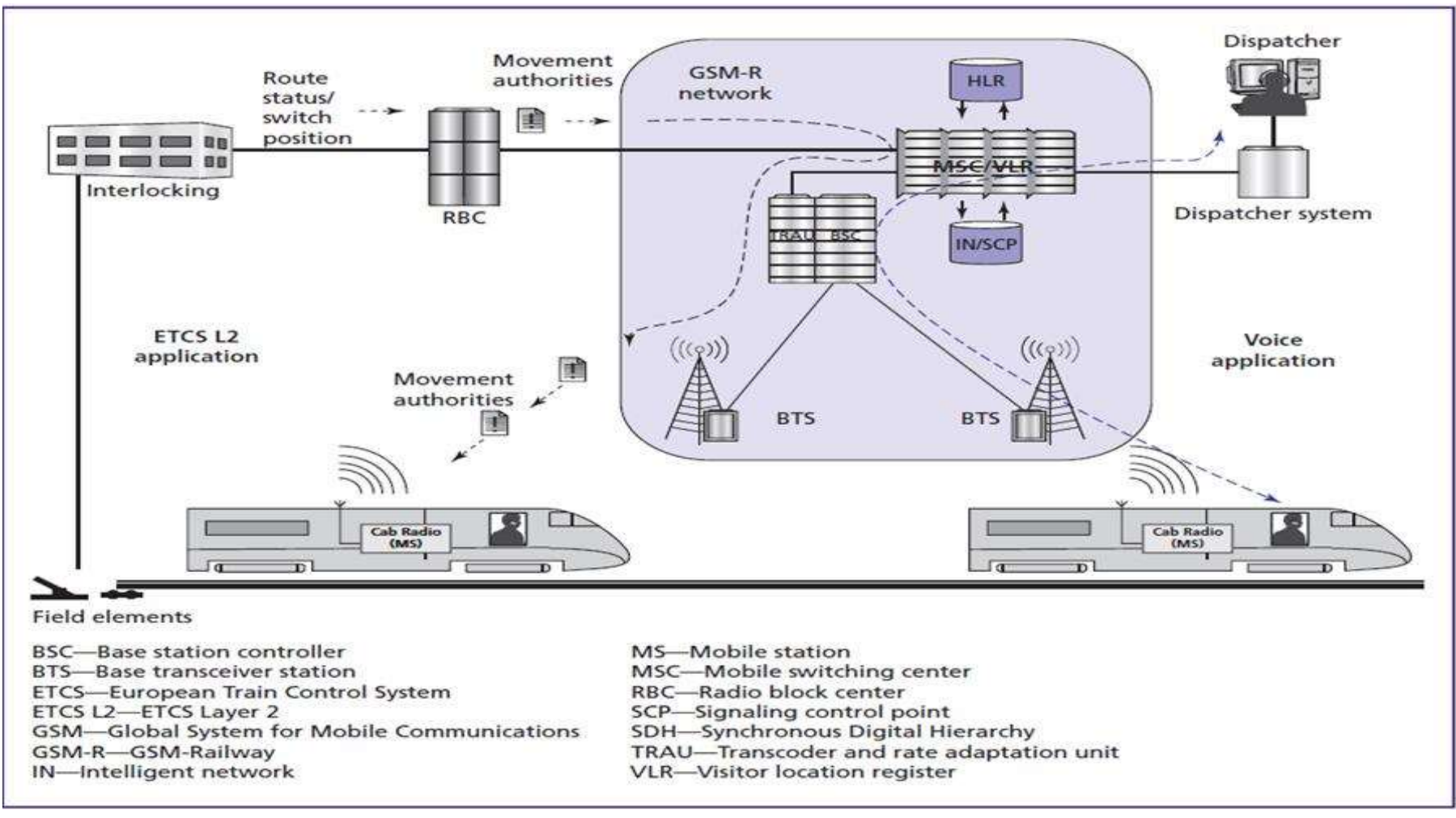

Figure 1. ETCS Level 2 voice and data communication [9] 
Based on the received messages, the embedded ETCS system calculates a dynamic train speed profile using location information as well as additional static data. When the GSM-R does not work, the train switches to the backup signaling system. Figure 1 shows the GSM system for providing voice and data services for ETCS level 2.

The basic services offered by GSM-R are: [10]

- Voice Group Call Service (VGCS) - where multiple people can be in the same call

- Voice Broadcast Service (VBS) - represents a group call with one person who is a speaker, the others in the call are listeners. This type of call is mainly used to broadcast recorded messages or for announcements.

- Multi-Level Enhanced Precedence and Pre-Emption (eMLPP) service - defines user priority and there are different priority levels

- The Railway Emergency Call (REC) is dedicated to urgency and is a dedicated group call with the number 299 that is established with an emergency level priority whose level is the highest possible priority 0 .

- Shunting Emergency Call (SEC) group call for staff involved in shunting, the call is established with an emergency level priority whose level is the highest possible priority 0 .

- Functional Addressing (FA) allows you to call a mobile station according to its function

- Location Dependent Addressing (LDA) is addressing method by information of the location the mobile station, where the call is forwarded to the nearest dispatcher.

The key advantages of the implementation of the GSM-R system, in accordance with EIRENE and MORANE specifications, are reflected in increased infrastructure capacity and safety of the railway network (implementation of GSM-R leads to multiple increases in line capacity resulting in higher workload, more passengers and cargo, and by higher traffic safety).

Then comes the unification of the existing radiocommunication system, and the interoperability of communications for European railway traffic is a goal to be achieved. The advantages of GSM-R implementation are interoperability and creation of conditions for upgrading the railway network with ERTMS / ETCS system (existing analog systems are obsolete and cannot be upgraded), simplification of communications compared to analog communication, fewer interruptions and train delays, shortening travel time, savings on signaling equipment, more efficient domestic and international transport lines and free movement of goods and people in Europe.

GSM-R has achieved great success not only in Europe where it is used daily on more than $100,000 \mathrm{~km}$ of railways but also in other parts of the world.[11] Although GSM-R is widely used, some suppliers of the supply industry have stated that obsolescence of GSM-R (eg due to aspects such as spare parts availability) can pose a risk in the long-distance, so an alternative system must be found. GSM-R technology has proven successful in terms of interoperability, performance and functionality, and meets the current operational needs of railways, but the allocated frequency spectrum has been shown to have several internal limitations, such as limited capacity and scalability for high-speed trains. [[12] The limited capabilities of GSM-R define an obstacle to the application of new services. The technology is not flexible enough in terms of flow and latency to provide advanced services and adjustments to new requirements in the future. As already mentioned, GSM-R is a secondgeneration telecommunications system, which means that it is far behind $4 \mathrm{G}$ and $5 \mathrm{G}$ technology.

\section{Overview of LTE-R}

\subsection{Development of LTE-R}

With the development of the high-speed railway, the technical requirements for the mobile communication system are growing. Since the implemented GSM-R communication system is based on the second generation GSM technology, it is necessary to adjust the communication methods to the new requirements on the railway market. In the future, the faults of GSM-R will become even more problematic as the demand for communication-based services will grow. Therefore, railways are looking for different technical and operational solutions for mobile communication systems.

Researchers, telecommunications system suppliers and railway companies have considered various technologies as candidates for the future railway communications network. The UIC General Assembly was held in Istanbul in 2008, announcing the possibility of applying Long Term Evolution LTE as the successor to GSM-R technology. Subsequently, in 2009, a technical report was published examining whether LTE communication systems would be applicable to an integrated rail wireless network. The conclusions were that LTE technology might be appropriate for the future, but further changes are needed. The first edition of the LTE standard was published almost 20 years after the GSM standard.

\subsection{The characteristics of LTE-R system}

LTE can operate in different bandwidths: $1.4 \mathrm{MHz}, 3$ $\mathrm{MHz}, 5 \mathrm{MHz}, 10 \mathrm{MHz}, 15 \mathrm{MHz}$, or $20 \mathrm{MHz}$ allowing network operators to flexibly manage their available radio spectrum. [13] Namely, the operator can share the radio band of the GSM network. One part would continue to be 
used via GSM, while the other part would be a new LTE network. The network operator could decide how much of the radio resources will allocate to LTE and how much to keep for GSM. This can be a very important feature for railways, as it means that the rail LTE network could gradually replace the GSM network. Table 2 presents some of the key parameters of the GSM-R system.

Table 2. Performance of LTE-R system [6] [14]

\begin{tabular}{cc}
\hline Parameter & GSM-R \\
\hline Frequency & $450 \mathrm{MHz}, 800 \mathrm{MHz}, 1.4$ \\
Bandwidth & $\mathrm{GHz}, 1.8 \mathrm{GHz}$ \\
Cell Range & $4.4-20 \mathrm{MHz}$ \\
Peak Spectral Efficiency & $2.55 \mathrm{bps} / \mathrm{Hz}$ \\
Data Transmission & Packet switching (UDP \\
Mobility & data) \\
Handover Success Rate & $\geq 900 \mathrm{~km} / \mathrm{h}$ \\
\hline
\end{tabular}

And basically, LTE is an efficient technology that offers high transmission capacity, high bandwidth and low latency. The use of network resources - especially limited radio resources - is optimized compared to GSMR. Transmission performance has been significantly improved in terms of flow and latency. Also, LTE offers standardized solutions for working together with GSM.

Compared to the GSM-R system, some special features of LTE for the railway that are recommended are:

- Transmission of control system information - LTE-R provides real-time control information transmission over a wireless communication network, with a delay of less than $50 \mathrm{~ms}$

- Real-time monitoring - LTE-R provides video recording or monitoring of the front of the track, cab, video monitoring of railway infrastructure (eg bridges and tunnels) to avoid natural disasters; and video crossover monitoring to detect freezing at low temperatures. This information are going to be shared with the center and with the real-time high-speed train, with a delay of but $300 \mathrm{~ms}$.

- Multimedia train dispatching - enable dispatch information (like text, data, voice, images, video, etc.) which influence dispatching efficiency. There are other functionalities such as voice channels, dynamic grouping, temporary group calls, short message exchanges, multimedia messaging, etc.

- Rail emergency communications - in the case of natural disasters, accidents or other emergencies, it is necessary to quickly establish communication between the accident site and rescue centers. Railway communication systems use a private network to ensure fast deployment and faster response.

- Internet of Things (IoT) devices connected to the Internet for tracking of fixed and mobile assets will grow in the daily life of the railway industry such as condition monitoring, fault prediction and maintenance routines and it will become more sophisticated to achieve increased capacity and reliability.[15]

In Figure 2. are shown the future services of the LTE$\mathrm{R}$ system based on the technical reports of the International Railway Union, China Railway, and the European Railway Agency.

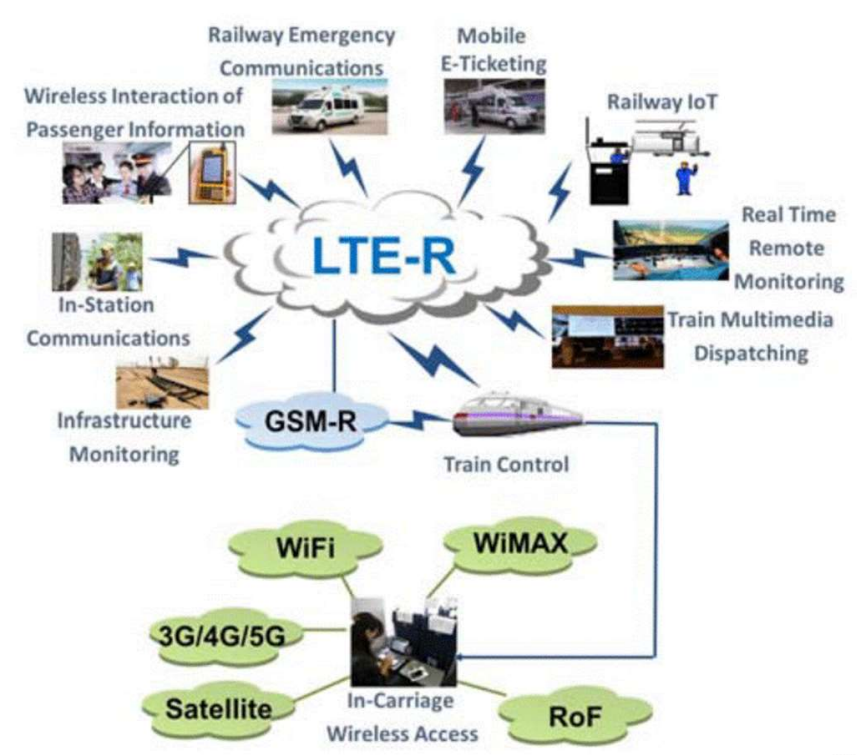

Figure 2. LTE-R services [8]

In future mobile communication systems, the main requirement will be information protection.[16] The transition from GSM-R to LTE-R could be divided into two phases, the first phase in which GSM-R and LTE-R would coexist, where GSM-R would be in charge of train control, and LTE-R for the security of data transmission, which is responsible for video surveillance and services for passengers and other data transmissions. In the second phase, LTE-R would provide more and more services and eventually, all communication would move to the LTE-R network. Whether LTE will become a rail communications technology depends on strategic choices such as network ownership and control model, possible network sharing with the public security sector or other industries, roaming in commercial networks, and frequency band allocation and timing. Yet, because of its many advantages, LTE must be considered as a good candidate for a future rail communications network, regardless of whether that communication network is based on one or more technologies. In the transition time 
from the GSM-R system to the new communication system, $5 \mathrm{G}$ as the globally dominant technology could replace LTE-R. [17] The UIC proposes that 5G be considered the preferred technology at the beginning of the introduction of a replacement system for GSM-R.

\section{Conclusion}

The process of adjustment to railway requirements has been a long process involving many participants: train operators, infrastructure operators, railway unions, national authorities and European authorities. The standardization process along with the process of convincing railway companies to use GSM-R technology took so many years that it was already obsolete at the time of implementation. The construction of a railway digital mobile communication network is a systematic project and is closely linked to railway dispatch communication, train control and operations management. Accordingly, mobile communication technologies should be fully exploited, combined with real rail transport needs and an integrated communication platform for rail transport. Taking into consideration given current trends in railways and telecommunications, it is likely that the future rail communications network will be based on multiple radio access technologies, high-speed communication networks will be heterogeneous with different networks and radio access technologies that can be accessed by hundreds of train users simultaneously. The rail transport sector is exploring digitalization to improve the user experience, increase efficiency in day-to-day operations, and maintain network security and data integrity. Fifth-generation mobile networks will have an increasing impact on the development of society and industry in the future. This impact will also be reflected on rail communication systems. Railway undertakings will be able to modernize existing networks and move to a new constitution that offers the highest safety, high operational efficiency.

\section{References}

[1] W. Myers and G. Warren-myers, "Survey of wireless communications applications in the railway industry," 2nd Int. Conf. Wirel. Broadband Ultra Wideband Commun., 2013.

[2] M. Sumiła and A. Miszkiewicz, "Analysis of the problem of interference of the public network operators to GSM-R," Commun. Comput. Inf. Sci., vol. 531, pp. 253-263, 2015, doi: 10.1007/978-3-319-24577-5 25.

[3] A. Sniady and J. Soler, "An overview of GSM-R technology and its shortcomings," 2012 12th Int. Conf. ITS Telecommun. ITST 2012, pp. 626-629, 2012, doi: 10.1109/ITST.2012.6425256.

[4] R. Chen, W. X. Long, G. Mao, and C. Li, "Development trends of mobile communication systems for railways," IEEE Commun. Surv. Tutorials, vol. 20, no. 4, pp. 3131-3141, 2018, doi:

\subsection{9/COMST.2018.2859347.}

[5] N. A. Korde and D. V. Rojatkar, "A Survey on Transferring Standards of GSM-R and Its Development in Railway Sector," Int. J. Sci. Res. Sci. Eng. Technol., vol. 2, no. 6, pp. 596-599, 2016.

[6] P. Fraga-Lamas, T. M. Fernández-Caramés, and L. Castedo, "Towards the internet of smart trains: A review on industrial IoT-connected railways," Sensors (Switzerland), vol. 17, no. 6, 2017, doi: 10.3390/s17061457.

[7] M. Heddebaut et al., "Towards a resilient railway communication network against electromagnetic attacks," Proc. TRA 2014, Transp. Res. Arena Conf., 2014.

[8] R. He, B. Ai, G. Wang, K. Guan, and Z. Zhong, "HighSpeed Railway Communications: from GSM-R to LTE-R,” 2020.

[9] K. C. Budka, J. G. Deshpande, T. L. Doumi, M. Madden, and T. Mew, "and Design Principles for Smart Grids," vol. 15, no. 2, pp. 205-227, 2010, doi: $10.1002 / \mathrm{bltj}$

[10] UIC CODE 951-16.0.0, "EIRENE - System Requirements Specification Version 16.0.0," no. December, 2015, [Online]. Available: https://uic.org/rail-system/gsm-r/.

[11] C. Prasad, "GSM-R to LTE for Railways in India," Proc. - 2018 IEEE Glob. Conf. Wirel. Comput. Networking, GCWCN 2018, pp. 16-19, 2019, doi: 10.1109/GCWCN.2018.8668584.

[12] A. Sniady and J. Soler, "Performance of LTE in high speed railway scenarios: Impact on transfer delay and integrity of ETCS messages," Lect. Notes Comput. Sci. (including Subser. Lect. Notes Artif. Intell. Lect. Notes Bioinformatics), vol. 7865 LNCS, pp. 211-222, 2013, doi: 10.1007/978-3-642-37974-1 17.

[13] K. I. Pedersen, F. Frederiksen, C. Rosa, H. Nguyen, L. G. U. Garcia, and Y. Wang, "Carrier aggregation for LTE-advanced: Functionality and performance aspects," IEEE Commun. Mag., vol. 49, no. 6, pp. 8995, 2011, doi: 10.1109/MCOM.2011.5783991.

[14] M. Sneps-Sneppe and D. Namiot, "Digital Railway and How to Move from GSM-R to LTE-R and 5G," Commun. Comput. Inf. Sci., vol. 1140 CCIS, pp. 392 402, 2020, doi: 10.1007/978-3-030-37436-5_34.

[15] O. Jo, Y. K. Kim, and J. Kim, "Internet of Things for Smart Railway: Feasibility and Applications," IEEE Internet Things J., vol. 5, no. 2, pp. 482-490, 2018, doi: 10.1109/JIOT.2017.2749401.

[16] Y. Wang, W. Zhang, X. Wang, W. Guo, M. K. Khan, and P. Fan, "Improving the Security of LTE-R for High-Speed Railway: From the Access Authentication View," IEEE Trans. Intell. Transp. Syst., pp. 1-15, 2020, doi: 10.1109/tits.2020.3024684.

[17] T. Chatelet, B. Domingo, W. Malfait, F. Ferrari, and P. Guido, "Evolution of Railway Radio Communication: System Definition," pp. 1-31, 2018, [Online]. Available:

https:/www.era.europa.eu/sites/default/files/activities/ docs/evolution_of_railway_radio_communication_syst em_definition.pdf. 